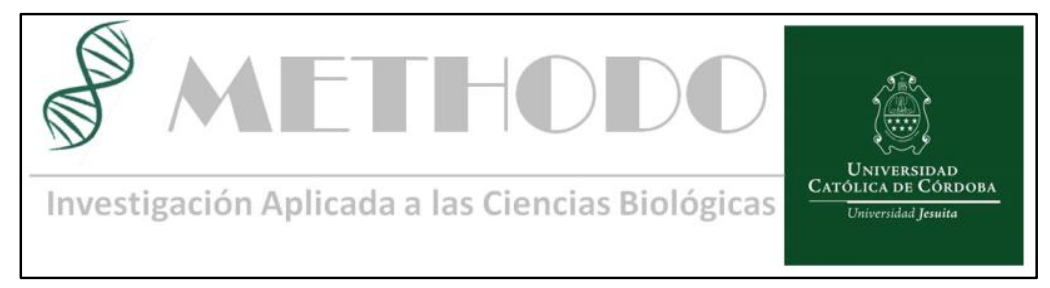

PRACTICA PROFESIONAL Articulo de opinión Methodo 2018;3(3):93-98

DOI: 10.22529/me.2018.3(3)09

Recibido 10 Ago 2018 | Aceptado 25 Ago 2018| Publicado 13 Sep 2018

\title{
Errores cognitivos de diagnóstico en medicina bucal
}

\section{Cognitive errors of diagnosis in oral medicine}

\author{
Dr. Eduardo David Piamonte ${ }^{1}$, Od Martín Brusa ${ }^{2}$, Dr. Ricardo Caciva ${ }^{3}$, Dr. René Luis Panico ${ }^{4}$ \\ ${ }^{1}$ Cátedra de Clínica Estomatológica, Carrera de Odontología, Facultad de Medicina, Universidad Católica de Córdoba. ${ }^{2}$ Cátedra de \\ Clínica Estomatológica, Carrera de Odontología, Facultad de Medicina, Universidad Católica de Córdoba. ${ }^{3}$ Cátedra de Clínica \\ Estomatológica, Carrera de Odontología, Facultad de Medicina, Universidad Católica de Córdoba. ${ }^{4}$ Cátedra de Clínica Estomatológica, \\ Carrera de Odontología, Facultad de Medicina, Universidad Católica de Córdoba.
}

\section{Resumen:}

La Medicina Bucal, como toda actividad humana, puede ser abordada desde dos sistemas de razonamiento, conocidos como Sistemas Cognitivos. El Sistema 1 es intuitivo, económico y rápido, mientras que el Sistema 2 es analítico, lento, costoso en esfuerzo, pero más confiable. Ambos sistemas interactúan entre sí, de manera que nuestra actividad de diagnóstico emplea a uno y otro. Los errores de diagnóstico en Medicina Bucal pueden basarse en falta de aplicación de reglas sistemáticas del sistema 2 , o en errores cognitivos al utilizar exclusivamente el sistema 1. Estos errores derivan generalmente en considerar falso un diagnóstico verdadero, o en considerar verdadero un diagnóstico falso. Existen estrategias para reducir estos errores cognitivos, pero el paso fundamental es conocer cómo pensamos y cuáles son los errores que podemos cometer. El análisis reflexivo de los errores cognitivos de diagnóstico (metacognición) permitiría transformarlos en oportunidades de crecimiento profesional y en una mejor atención para nuestros pacientes.

\footnotetext{
Abstract:

Oral Medicine, like all human activity, can be approached from two systems of reasoning, known as Cognitive Systems. System 1 is intuitive, economical and fast, while System 2 is analytical, slow, costly in effort, but more reliable. Both systems interact with each other, so that in our diagnostic activity we use both. Diagnosis errors in Oral Medicine can be based on the lack of application of systematic rules of the system 2 , or on cognitive errors when the system 1 is used
}

exclusively. These errors derive generally in false considering a true diagnosis, or in considering true a diagnosis false. There are strategies to reduce these cognitive errors, but the fundamental step is to know how we think and what are the mistakes we can make. The reflexive analysis of cognitive errors of diagnosis (metacognition) would allow transforming them into opportunities for professional growth and better care for our patients.

\section{Introducción:}

El diagnóstico en medicina bucal es un elemento fundamental en el proceso de conseguir la curación del paciente. Un diagnóstico incorrecto puede significar una pérdida de oportunidad de tratar adecuadamente la enfermedad, o inclusive, puede generar daños en el paciente. Las especialidades de la salud en las que el examen visual tiene una influencia mayor en el diagnóstico, como el caso de la medicina bucal, suelen tener menor porcentaje de error que otras especialidades médicas en las que se debe recurrir a otros medios de recolección de datos ${ }^{1}$.

Sin embargo, como toda actividad humana, el diagnóstico en medicina bucal está expuesto a errores, ya sea porque no se realizó diagnóstico, porque se hizo un diagnóstico incorrecto, o porque el diagnóstico fue realizado tardíamente. Los errores en el proceso de diagnóstico pueden a su vez obedecer a distintas causas. Puede haber errores sin culpa, cuando se debe a una manifestación poco habitual o extraordinaria de una enfermedad. Puede haber errores atribuibles al

Revista Methodo: Investigación Aplicada a las Ciencias Biológicas. Universidad Católica de Córdoba. Jacinto Ríos 571 Bo Gral. Paz. X5004FXS. Córdoba. Argentina. Tel.: (54) 3514517299 / Correo: methodo@ucc.edu.ar / Web: methodo.ucc.edu.ar | PRACTICA PROFESIONAL Articulo de opinión Methodo 2018;3(3):93-98 
sistema de salud, debido a la demora burocrática o a deficiencias en la comunicación intra e interinstitucionales. Y puede haber también errores cognitivos, debidos no tanto a cuánto conoce el profesional, sino a cómo es la forma de pensar y de razonar cuando debe establecer un diagnóstico. ${ }^{2-3}$ Para entender por qué los profesionales de la salud cometemos errores en el diagnóstico, es necesario entender cómo son los mecanismos cognitivos para tomar decisiones. En todos los aspectos de la vida el ser humano toma decisiones de acuerdo a dos sistemas de pensamiento, que se interrelacionan permanentemente. Existe un sistema intuitivo, que permite tomar decisiones rápidas con bajo costo de tiempo y energía, conocido como sistema 1; y existe otro sistema, analítico, conocido como sistema 2, que permite tomar decisiones más precisas, pero con mayor costo de tiempo y energía. ${ }^{4-5}$

El Sistema 1, basado en experiencias previas, opera a través de un reconocimiento holístico de una situación como alguna otra encontrada previamente, la cual trae a la memoria un esquema que brinda una solución. El Sistema 2, por otra parte, depende de la aplicación de reglas de diagnóstico que asocian ciertos síntomas o signos con la mayor probabilidad de que ocurra una enfermedad en particular. Ambos sistemas conviven y se retroalimentan. El sistema 1 puede ser manejado por el sistema 2 si detecta algún mal funcionamiento. La educación y el encuentro repetido con una situación terminan convirtiendo ese evento en particular en una respuesta tipo 1. Esto ocurre al empezar a diagnosticar lesiones de mucosa bucal. Las primeras veces, tal como sucede en ámbitos educativos, el diagnóstico es realizado por un sistema tipo 2. Posteriormente los profesionales de la salud, debido a la mayor experiencia que van adquiriendo y a la necesidad de mayor eficiencia energética, tienden a emplear el sistema 1, automático, en la cual la sola visión de la lesión permite hacer el diagnóstico ${ }^{6-7}$ (figura $1)$.

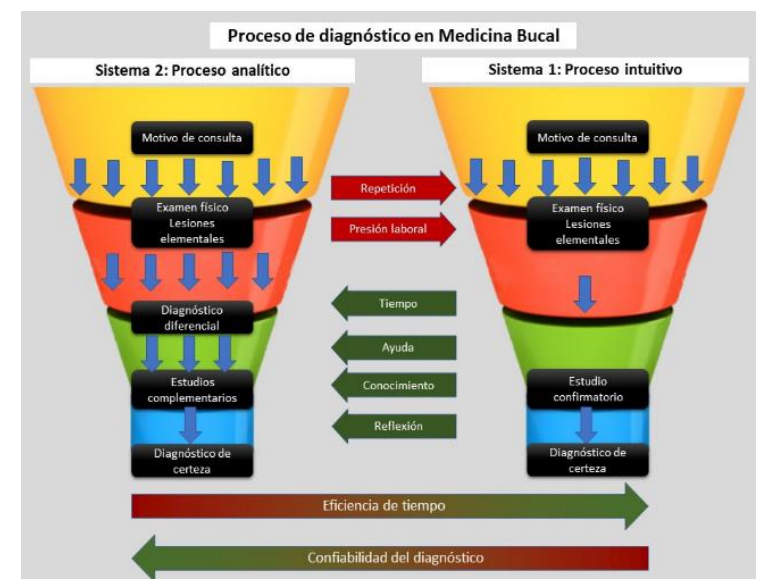

Figura 1. Diferencias en el proceso de diagnóstico en medicina bucal, según sistema cognitivo

\section{Errores cognitivos}

En ambos sistemas cognitivos puede haber errores, aunque en el sistema 2 es más fácil detectar cuál ha sido la causa. En el sistema 2 los errores obedecen generalmente a un mal empleo de los procedimientos de diagnóstico, a una mala interpretación de los resultados de estudios complementarios o a un contexto laboral de sobreexigencia del profesional. En el sistema 1, el empleo de reglas o vías rápidas de diagnóstico, permiten al profesional experimentado resolver la amplia mayoría de los casos que atienden. Sin embargo, esto genera sesgos que pueden derivar en errores cognitivos relevantes en la evolución del paciente al considerar falso un diagnóstico verdadero, o considerar verdadero un diagnóstico falso. $^{8}$

Entre otros, los errores cognitivos más frecuentes en el sistema 1 son los siguientes ${ }^{6-9-12}$

Error de disponibilidad: aceptar un diagnóstico debido a la facilidad para recordar un caso similar, más que en la probabilidad o prevalencia. También por falta de conocimientos suficientes. (figura 2) Ej.: si hace poco hemos visto un paciente con un cáncer en encía, al concurrir otro paciente con un tumor en encía pensamos exclusivamente en algún cáncer bucal y no en un granuloma teleangiectásico, que es el tumor más frecuente en encía. $\mathrm{O}$, por otra parte, también podemos pensar sólo en granuloma teleangiesctásico porque es el más frecuente y el que más conocemos, y no considerar la posibilidad, aunque sea menor, de un proceso maligno.

Error de representatividad: resolver un diagnóstico por comparación de las características más relevantes de un nuevo caso con una enfermedad conocida, dejando de tener en cuenta que las enfermedades pueden presentar manifestaciones excepcionales o atípicas. Ej: el chancro de sífilis

Revista Methodo: Investigación Aplicada a las Ciencias Biológicas. Universidad Católica de Córdoba. Jacinto Ríos 571 Bo Gral. Paz. X5004FXS. Córdoba. Argentina. Tel.: (54) 3514517299 / Correo: methodo@ucc.edu.ar / Web: methodo.ucc.edu.ar | PRACTICA PROFESIONAL Artículo de opinión Methodo 2018;3(3):93-98 
primaria es una erosión o úlcera poco profunda que asienta generalmente en labio o lengua. Un chancro "atípico" en zona posterior de la boca se manifiesta con una lesión ulcerovegetante, muy agresiva, que frecuentemente es confundido con un tumor maligno, el cual suele manifestarse como una lesión ulcerovegetante.

Cierre prematuro: conformarse con el primer hallazgo, atribuyendo la enfermedad del paciente a este fenómeno y no continuar la búsqueda si así lo ameritan los síntomas, por lo que no se consideran comorbilidades, complicaciones o diagnósticos adicionales. (figura 4) Ej: frente a una lesión ulcerovegetante, el informe de una biopsia de rutina indica inflamación inespecífica y ausencia de malignidad. La lesión resuelve espontáneamente y le informamos al paciente que no es una enfermedad grave, que fue una infección circunstancial. Pero no consideramos que esa lesión autorresolutiva era un chancro de sífilis.

Error de anclaje: no ajustar el diagnóstico inicial cuando información adicional, como estudios de laboratorio, modificación del cuadro clínico, o respuesta no esperada frente al tratamiento, sugieren reevaluar el diagnóstico. (figura 3) Ej: frente a un aumento de volumen en encía, que no resuelve con antibióticos ni con tratamiento periodontal, insistimos con otro antibiótico más potente, cuando la enfermedad no es realmente de origen infeccioso sino de origen tumoral.

Error de contexto: llegar a conclusiones distintas de acuerdo a cómo la información es presentada, por información colateral. Ej: un colega nos deriva un paciente haciéndonos el comentario de que es un paciente con alguna alteración psicológica. Apenas ingresa al consultorio, el paciente nos relata de manera angustiosa que le arde intensamente la lengua, e inmediatamente pensamos en un diagnóstico de síndrome de ardor bucal, que tiene una base emocional importante. Sin embargo, el paciente bien podría tener una candidiasis aguda, un secundarismo sifilítico o una enfermedad ampollar.

Confirmación sesgada: dar importancia a evidencia que coincide con la hipótesis de diagnóstico, y rechazar o desvalorizar la evidencia que se contrapone a la misma. Ej: un paciente presenta una leucoplasia con áreas rojas, y nuestro diagnóstico presuntivo es de carcinoma secundario a la leucoplasia. Sin embargo, esa zona roja puede obedecer a un área traumatizada o sobre infectada por candidiasis, factores que deben ser determinados y controlados primero antes de definir un diagnóstico.

Error de agregación o falacia ecológica: creer que los datos obtenidos de promedios grupales son aplicables a personas individuales. Ej: los factores de riesgo más importantes de cáncer bucal son el consumo de tabaco y de alcohol. En un paciente no fumador y no bebedor se puede desestimar el diagnóstico de cáncer bucal, aunque sea verdadero. Falacia del jugador: creer que, si el mismo diagnóstico se realizó en varios sucesivos pacientes, es menos probable que ese diagnóstico ocurra en el siguiente paciente. Ej.: en una misma mañana se diagnostica dos pacientes con cáncer bucal. El profesional considera "imposible" que el tercer paciente tenga también cáncer bucal.

Obediencia ciega: creer sin cuestionamientos a la palabra de expertos, situación a veces mencionada como "medicina basada en la eminencia" (no en la evidencia). Ej.: al concurrir a un curso dictado por un eminente profesor de Medicina Bucal, se imparten ciertos conocimientos o conceptos, aún sin la debida evidencia científica. Por ejemplo, que la variedad histológica de un tumor anexial varía si está hacia la línea media o lateralizado. El correcto diagnóstico se realiza con biopsia, y no por la localización del tumor.

\section{Estrategias para evitar errores cognitivos}

Para mejorar el proceso cognitivo del diagnóstico, la conciencia y la comprensión de los propios procesos de pensamiento, denominado metacognición, permite establecer diversas estrategias que permiten disminuir errores como los descriptos. Aunque, básicamente, todas estas estrategias procuran hacer más conscientemente el razonamiento, para convertir el sistema cognitivo 1 en un sistema cognitivo más aproximado al sistema 2. Esto no significa que todos y cada uno de los procesos intuitivos deban ser convertidos en analíticos, ya que esto sería desgastante e impráctico debido a la carga de tiempo y de esfuerzo cognitivo que implicaría, e inclusive podría generar parálisis de los procesos de diagnósticos aún en casos en donde es urgente actuar con el tratamiento

A largo plazo, la mejor manera de mejorar la calidad del diagnóstico es convertirse en un experto en un área específica. Por definición, los expertos cometen menor cantidad de errores (o más seguramente, los han hecho todos antes). La excelencia en el sistema 2 demanda un conocimiento extenso y actualizado, especializado en pensamiento crítico, basado en evidencia científica y en una experiencia clínica amplia y profunda. Todo esto lleva tiempo y evoluciona a través de toda una vida de trabajo6.

Mientras tanto, ¿qué podemos hacer? Debido a que varios errores de diagnóstico asientan sobre deficiencias propias del sistema cognitivo 1, aprender sobre estas y emplear estrategias para disminuir estos errores son vías potencialmente efectivas para disminuir los errores de diagnóstico.

Revista Methodo: Investigación Aplicada a las Ciencias Biológicas. Universidad Católica de Córdoba. Jacinto Ríos 571 Bo Gral. Paz. X5004FXS. Córdoba. Argentina. Tel.: (54) 3514517299 / Correo: methodo@ucc.edu.ar / Web: methodo.ucc.edu.ar | PRACTICA PROFESIONAL Artículo de opinión Methodo 2018;3(3):93-98 
Probablemente el mejor consejo sea simplemente tomarse un tiempo para hacer el diagnóstico. Reflexionar sobre cada caso permite al Sistema 2 revisar adecuadamente el proceso de diagnóstico y poder identificar inconsistencias.

Como los errores de contexto y de cierre prematuro son dos de los errores más comunes del sistema 1 , las estrategias para promover la consideración de otras posibilidades diagnósticas son recomendables. El antídoto universal es simple, preguntándose cuál otro diagnóstico podría ser. Completar el diagnóstico diferencial es otra vía para evitar enfoques inadecuados.

Cuando unimos todos los síntomas y signos que encontramos en un paciente, sobre todo partiendo de las lesiones elementales que presenta en su boca, podemos proponer alternativas de diagnóstico que incluyan estas manifestaciones clínicas. Con ello hacemos el diagnóstico comparando el cuadro clínico que presenta el paciente con el de estas enfermedades: qué tiene o qué no tiene de una o de otra, cuáles son las diferencias y semejanzas con cada afección, a cuál se parece más el cuadro del paciente y a cuáles menos. El paciente no siempre manifiesta todos los síntomas y signos de la afección, y otras enfermedades pueden tener síntomas y signos similares a los del paciente. Por ello, el diagnóstico se realiza por la mayor semejanza del cuadro clínico del paciente con el descrito para determinada enfermedad. El requerimiento de los diagnósticos diferenciales podría actuar como un disparador para iniciar la estructura del razonamiento. El diagnóstico diferencial sirve como filtro de datos y genera gran objetividad para refutar hipótesis de diagnóstico alternativas. ${ }^{13}$

A fines prácticos, los medios de disminuir los errores cognitivos en el diagnóstico pueden resumirse en cinco puntos. ${ }^{6-9-10-14-15}$

1.Sea reflexivo, tómese un tiempo para el diagnóstico: Existen situaciones en las que hay alto riesgo de error de diagnóstico. Para evitarlas es conveniente detenerse el tiempo suficiente para realizarse sistemáticamente las siguientes preguntas: ¿Hay otros diagnósticos que debo considerar? ¿Debo aceptar el primer diagnóstico que se me ocurrió? ¿El diagnóstico me fue sugerido por el paciente o por otro profesional? ¿Me falta algún dato para evaluar el caso? ¿Hay aspectos del paciente que no encajan con el diagnóstico? ¿Estuve distraído, o fui interrumpido cuando realicé el diagnóstico? ¿El paciente tiene alguna relación afectiva positiva o negativa conmigo, que pueda influir en mi decisión?

2.Obtenga su propia y completa historia clínica, escuchando y examinando detenida y minuciosamente a los pacientes. Los avances tecnológicos han conducido a una preponderancia de las pruebas de laboratorio como métodos de diagnóstico. Aun así, el examen clínico sigue siendo crucial en la evaluación de la mayoría de los pacientes. Este proceso implica considerar todos los datos, clínicos y complementarios, y no exámenes aislados. Esto contribuye fundamentalmente a reducir los errores por cierre prematuro, al abandonar el proceso de diagnóstico cuando alguna prueba individual coincida con el cuadro clínico, sin considerar la totalidad de las manifestaciones. Pregúntese también porqué el paciente presenta determinada característica. No se trata de hacer un caso complejo de un caso simple, sino reforzar la importancia de examinar todos los casos en detalle.

3. Conocer cómo funciona nuestro razonamiento, cuáles son los errores cognitivos y cómo podemos evitarlos. Prestar atención a datos que no coincidan con la hipótesis diagnóstica. El escepticismo sobre una fuerte hipótesis de diagnóstico promueve el minucioso análisis de los datos disponibles que dicha hipótesis de diagnóstico no puede explicar. 4.Promueva el uso de un abordaje sistemático, inclusive para problemas comunes: no confíe en su intuición, porque, aunque las enfermedades sean frecuentes, el abordaje de diagnóstico debe hacerse siempre de manera sistemática, entre otras formas a través del planteamiento de los diagnósticos diferenciales. Tómese un tiempo para considerar todas las opciones de diagnóstico y para revisar la evidencia que apoya a un determinado diagnóstico, haciendo preguntas apropiadas, realizando exámenes físicos adicionales y solicitando estudios complementarios. También es conveniente plantear cuál sería el peor escenario de diagnóstico, cuáles diagnósticos no debo dejar de considerar, o qué otra enfermedad podría ser. Esta técnica tiene el potencial de incrementar el diagnóstico de enfermedades más graves, pero menos frecuentes.

5.Déjese ayudar: Desde el más novato hasta el más experimentado pueden cometer errores. La ventaja del más experimentado es que ya los cometió, y puede conocer cuáles fueron las causas por las que los cometió. Más allá de que uno confíe en sus propios recursos, pedir ayuda es otro enfoque que evita errores diagnósticos en algunos casos particulares. Comentar esos casos con colegas o en foros de discusión puede ayudar tanto al que cometió el error como a sus interlocutores a estar más alerta ante situaciones similares. En este sentido, actualmente cobran suma relevancia las interconsultas por telemedicina. Las segundas opiniones permiten enfocar el problema desde otras perspectivas, detectar datos que se pasaron por alto y plantear adicionales alternativas de diagnóstico, entre otras ventajas. Dejarse ayudar incluye también mantener contacto directo con los

Revista Methodo: Investigación Aplicada a las Ciencias Biológicas. Universidad Católica de Córdoba. 
profesionales que realizan pruebas diagnósticas, para seleccionar los métodos de diagnóstico más apropiados; permitir a sus colegas hacerle saber si el diagnóstico que usted realizó ha cambiado, e inclusive hacer a su paciente su socio en el diagnóstico, asegurándose que ellos sepan cómo hacerle saber a usted si los síntomas cambian o persisten. Admitir los propios errores y las propias limitaciones, inclusive públicamente, es fundamentalmente una oportunidad para mejorar.

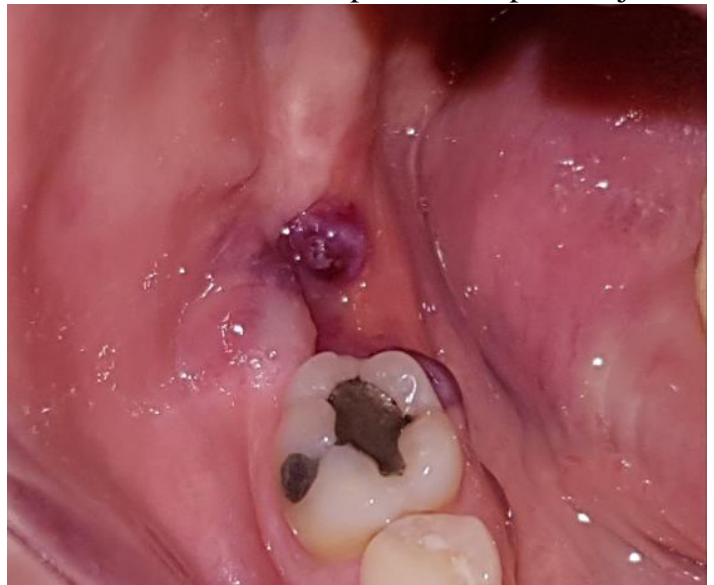

Figura 2. lesión tumoral en encía que fue extirpada quirúrgicamente con un diagnóstico presuntivo de granuloma teleangiectásico. No se consideró la posibilidad de que sea otra enfermedad menos frecuente (error de disponibilidad). La lesión recidivó y mediante biopsia y análisis de sangre se reveló un diagnóstico de sarcoma de Kaposi en un paciente HIV+.

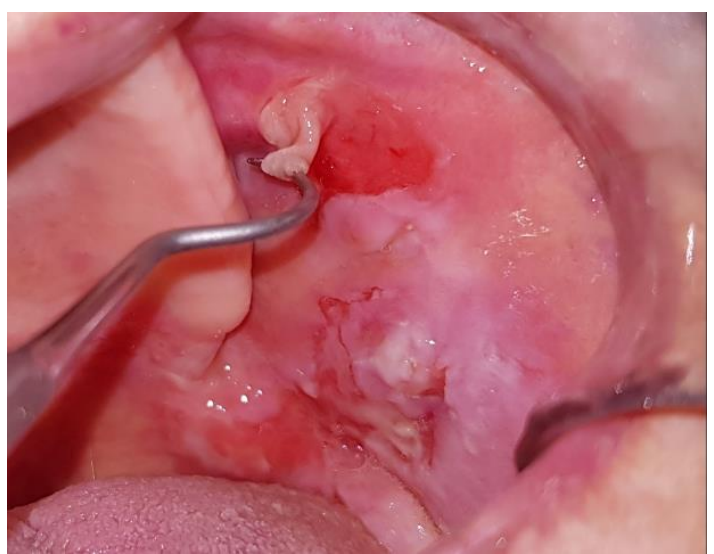

Figura 3. Paciente con múltiples lesiones blancas y rojas en la mucosa bucal, de 6 meses de evolución. Diagnosticada como candidiasis recibió sucesivos tratamientos con antifúngicos y antisépticos (error de anclaje). El análisis sistemático del caso permite definir la presencia de lesiones compatibles con ampollas con techo roto, lo que orienta hacia un diagnóstico de enfermedad ampollar como pénfigo o penfigoide, y no hacia candidiasis.

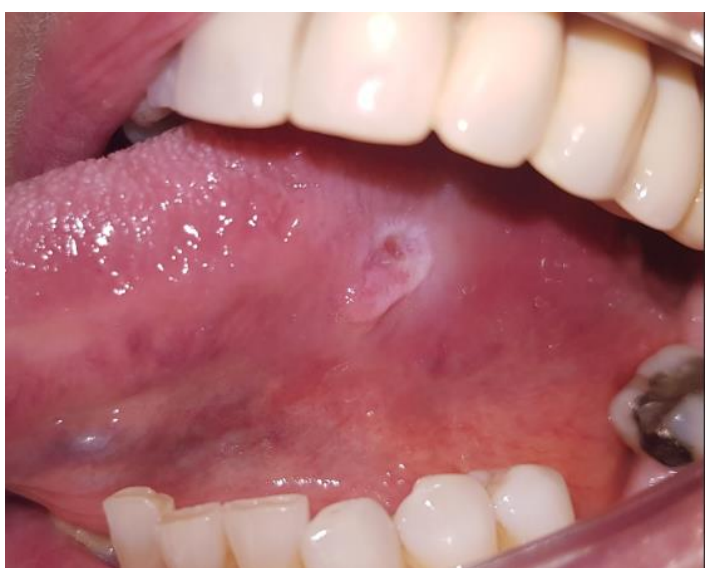

Figura 4. úlcera de 2 meses de evolución en borde de lengua, con dolor leve y a veces irradiado al oído. poco dolorosa, pero siente sensibilidad en la zona y que en ocasiones siente dolor irradiado al oído. Al observar la relación de contacto entre la úlcera y el molar inferior lingualizado se establece el diagnóstico de úlcera traumática crónica, se indica la extracción del molar y no se realizan más estudios (cierre prematuro). La lesión, que no desapareció, fue posteriormente diagnosticada como carcinoma de células escamosas, basado en biopsia y anatomía patológica. Este error de cierre prematuro podría haberse prevenido si se hubiera considerado analizar sistemáticamente el caso, teniendo en cuenta que, ante una úlcera crónica en mucosa bucal, el carcinoma de células escamosas siempre debe considerarse como un posible diagnóstico diferencial.

\section{Conclusión:}

Los errores de diagnóstico en Medicina Bucal pueden basarse en la falta de aplicación de reglas sistemáticas de diagnóstico, o en errores cognitivos, de razonamiento, cuando no se aplican reglas sistemáticas sino atajos de razonamiento. Existen estrategias para reducir estos errores cognitivos, pero el paso fundamental es conocer cómo razonamos y cuáles son los errores que podemos cometer. El análisis reflexivo de los errores cognitivos de diagnóstico permitiría transformarlos en oportunidades de crecimiento profesional y en una mejor atención para nuestros pacientes.

Revista Methodo: Investigación Aplicada a las Ciencias Biológicas. Universidad Católica de Córdoba. Jacinto Ríos 571 Bo Gral. Paz. X5004FXS. Córdoba. Argentina. Tel.: (54) 3514517299 / Correo: methodo@ucc.edu.ar / Web: methodo.ucc.edu.ar | PRACTICA PROFESIONAL Artículo de opinión Methodo 2018;3(3):93-98 


\section{Bibliografía:}

1. Berner ES, Graber ML. Overconfidence as a Cause of Diagnostic Error in Medicine. Am J Med 2008;121(5): S2-23.

2. Aspiazú B, Hernández Azcuy O, Bayard B, Moreno Rodríguez M. Errores cometidos por residentes de Medicina Interna en la entrevista médica bajo observación directa. Rev Cuba Med Mil 2002;31(2):104-9.

3. Graber M, Gordon R, Franklin N. Reducingdiagnosticerrors in medicine: ¿what'sthegoal?AcadMed J Assoc Am MedColl 2002;77(10):981-92.

4. Stanovich KE. Whoisrational?: Studies of individual differences in reasoning. Mahwah, NJ, US: Lawrence ErlbaumAssociatesPublishers; 1999. xvi, 296.

5. Stanovich KE, West RF. Individual differences in reasoning: implicationsfortherationality debate? BehavBrainSci 2000;23(5):645-65; discussion 665-726.

6. ClinicalExcellenceComission. Diagnostic Error: LearningResourceforClinicians. ClinicalExcellenceComission; 2015.

7. Croskerry P. A universal model of diagnosticreasoning. AcadMed J Assoc Am MedColl 2009;84(8):1022-8.

8. Cernadas C, M J. Errores de diagnóstico en la práctica médica. Arch Argent Pediatría 2015;113(3):194-5.

9. Wainstein E. Theheuristics of reaching a diagnosis. RevMedChil 2009;137(12):163641.

10. Croskerry P. Theimportance of cognitiveerrors in diagnosis and strategies to minimizethem. AcadMed J Assoc Am MedColl 2003;78(8):775-80.

11. Nendaz M, Perrier A. Diagnosticerrors and flaws in clinicalreasoning: mechanisms and prevention in practice. SwissMedWkly 2012;142: w13706.

12. Lawson AE, Daniel ES. Inferences of clinicaldiagnostic reasoning and diagnostic error. J BiomedInform 2011;44(3):402-12.

13. Maude Jason. Differential diagnosis: thekey to reducing diagnosis error, measuring diagnosis and a mechanism to reduce healthcare costs. Diagnosis 2014;1(1):107.
14. Trowbridge RL. Twelve tips for teaching avoidance of diagnosticerrors. MedTeach 2008;30(5):496-500.

15. Croskerry $\mathrm{P}$, Singhal G, Mamede $\mathrm{S}$. Cognitivedebiasing 2: impediments to and strategiesforchange. BMJ QualSaf 2013;22 Suppl 2: ii65-72.

\section{Palabras claves:}

MEDICINA BUCAL, ERRORES DE
DIAGNÓSTICO, METACOGNICIÓN

\section{Keywords:}

ORAL MEDICINE, DIAGNOSTICERRORS, METACOGNITION

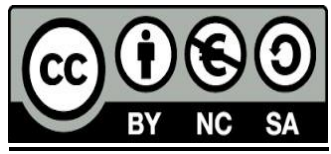

Revista Methodo: Investigación Aplicada a las Ciencias Biológicas. Universidad Católica de Córdoba. Jacinto Ríos 571 Bo Gral. Paz. X5004FXS. Córdoba. Argentina. Tel.: (54) 3514517299 / Correo: methodo@ucc.edu.ar / Web: methodo.ucc.edu.ar | PRACTICA PROFESIONAL Artículo de opinión Methodo 2018;3(3):93-98 